\title{
Essais
}

Revue interdisciplinaire d'Humanités

$7 \mid 2015$

Normes communiquées, normes communicantes

\section{La maternité dans les séries familiales françaises : entre consensus et résistances}

Sarah Lécossais

\section{OpenEdition}

1 Journals

Édition électronique

URL : http://journals.openedition.org/essais/6183

DOI : 10.4000/essais. 6183

ISSN : 2276-0970

Éditeur

École doctorale Montaigne Humanités

Édition imprimée

Date de publication : 1 décembre 2015

Pagination : 30-47

ISBN : 978-2-9544269-6-9

ISSN : 2417-4211

Référence électronique

Sarah Lécossais, «La maternité dans les séries familiales françaises : entre consensus et

résistances », Essais [En ligne], 7 | 2015, mis en ligne le 26 février 2021, consulté le 27 février 2021.

URL : http://journals.openedition.org/essais/6183 ; DOI : https://doi.org/10.4000/essais.6183 


\section{La maternité dans les séries familiales françaises : entre consensus et résistances}

\section{Sarah Lécossais}

En tant qu' " espaces de représentation ", les médias constituent, selon Stuart Hall, un "champ structuré en domination " au sein duquel des représentations dominantes cherchent à s'imposer comme «allant de soi $»^{1}$. Pour autant, cette recherche du consensus engendre en retour des contradictions, des représentations marginales et donc des résistances. C'est l'ambivalence des productions médiatiques que nous souhaiterions analyser ici, en nous saisissant des représentations fictionnelles de la maternité à la télévision.

À la croisée des sciences de l'information et de la communication, de la sociologie des médias, des culturalstudies et des genderstudies, notre travail s'inscrit tout d'abord dans la lignée des travaux sur les représentations médiatiques. Nous emboîtons ainsi le pas de chercheuses comme Simone Bonnafous ${ }^{2}$, Michèle Mattelart ${ }^{3}$, Marlène Coulomb-Gully ${ }^{4}$ ou Marie-Joseph Bertini ${ }^{5}$ qui ont ouvert la voie et démontré et réaffirmé ${ }^{6}$ la nécessité de travailler sur les femmes, le genre et les médias. Au sein de la discipline des sciences de l'information et de la communication, les représentations médiatiques des femmes et du genre ou de problématiques adjacentes ont avant tout été analysées à travers les médias

1 Stuart Hall, «La culture, les médias et l'“effet idéologique” ", in Hervé Glévarec, Éric Macé et Éric Maigret (éd.), Anthologie des Cultural Studies, Paris, INA, 2009, p. 45.

2 Simone Bonnafous, " "Femme politique" : une question de genre ?", Réseaux, vol. 4, n 120, 2003, p. 119-145.

3 Michèle Mattelart, "Femmes et medias. Retour sur une problématique ", Réseaux, vol. 4, $\mathrm{n}^{\circ} 120,2003$, p. 23-51.

4 Marlène Coulomb-Gully, "Féminin/masculin : question(s) pour les SIC. Réflexions théoriques et méthodologiques ", Questions de Communication, n 17, 2010, p. 169-194.

5 Marie-Joseph Bertini, "Langage et pouvoir : la femme dans les médias (1995-2002) ", Communication et langages, $\mathrm{n}^{\circ} 152$, juin 2007, p. 3-22.

6 Marlène Coulomb-Gully, «Inoculer le Genre. Le Genre et les SHS : une méthodologie traversière ", Revue Française des Sciences de l'Information et de la Communication, n 4, 2014 [en ligne] : http://rfsic.revues.org/837 [consulté le 03/05/2014]. 
d'information $^{7}$, de divertissement ${ }^{8}$ ou encore la publicité ${ }^{9}$, au détriment des programmes fictionnels, et plus spécifiquement des séries télévisées. En études cinématographiques ou en sociologie des médias, les travaux de Geneviève Sellier sur les séries policières et de Sabine Chalvon-Demersay sur la fiction télévisée constituent des références solides sur lesquelles appuyer notre propos. La première montre que les productions culturelles - les séries télévisées comme le cinéma - "participent à la construction des normes sexuées, à la "fabrique du genre" particulière à chaque société et à chaque période $"^{10}$. D'un point de vue méthodologique, la seconde nous invite à considérer les personnages " comme s'ils étaient de véritables acteurs sociaux ", et à les analyser en regard de la société dans laquelle ils prennent vie. Les séries télévisées françaises méritent que l'on s'y arrête dans la mesure où, si l'on suit Éric Macé, elles constituent, en tant que programmes de télévision, des doubles télévisuels du monde social et permettent d'accéder à la "manière dont se disent elles-mêmes les sociétés ${ }^{11}$. Les séries nous informent sur le social ${ }^{12}$, ne serait-ce que parce que, comme l'écrit Marlène Coulomb-Gully à propos de la fiction télévisée : «l'imaginaire $[\ldots]$ ne constitue pas un continent autonome car, toujours, la fiction nous parle du réel $»^{13}$.

L'objectif de cet article est de mettre en lumière les normes relatives à la maternité telles qu'elles sont distillées dans les séries télévisées françaises, diffusées sur les principales chaînes hertziennes entre 1992 et $2012^{14}$. Notre hypothèse

7 Voir Aurélie Olivesi, Implicitement sexiste? Genre, politique et discours journalistique, Toulouse, Presses Universitaires du Mirail, 2012 ; Virginie Julliard et Aurélie Olivesi, "La presse écrite d'information : un média aveugle à la question du Genre. Reconduction des stéréotypes et naturalisation des rapports de sexe ", Sciences de la société, n ${ }^{\circ}$ 83, 2012, p. 36-53 et Virginie Julliard, De la presse à Internet. La parité en question, Paris, Lavoisier, 2012.

8 Nelly Quemener, Le Pouvoir de l'humour, Paris, Armand Colin, 2014.

9 Stéphanie Kunert, Publicité, genre et stéréotype, Fontenay-le-Comte, Lussaud, 2014.

10 Noël Burch et Geneviève Sellier, Le Cinéma au prisme des rapports de sexe, Paris, Vrin, 2009, p. 10.

11 Éric Macé, Les Imaginaires médiatiques. Une sociologie postcritique des médias, Paris, Éd. Amsterdam, 2006, p. 11.

12 Sabine Chalvon-Demersay, "Une société élective. Scénarios pour un monde de relations choisies ", Terrain, n² 27, 1996, p. 81-100.

13 Marlène Coulomb-Gully, Présidente : le grand déf. Femmes, politique et médias, Paris, Payot, 2012, p. 16.

14 Notre corpus se compose des séries de production française - tous formats et genres confondus centrées sur une famille (Une Famille formidable sur TF1 depuis 1992, Famille d'accueil sur France 3 depuis 2001 ou encore Ma femme, ma fille, deux bébés sur M6 depuis 2010) ou plusieurs familles (Fais pas ci, fais pas ça sur France 2 depuis 2007). Nous y ajoutons les séries dont l'héroïne ou les héroïnes sont des mères de famille (Hard sur Canal + en 2008 et 2011, Jeu de dames sur France 3 en 2012). En tout, notre corpus comprend une vingtaine de séries. L'un des critères de constitution est la mise en scène de la vie quotidienne et domestique des personnages, ce qui exclut du corpus les séries policières ou judiciaires à héroïnes récurrentes qui se focalisent sur le monde du travail. Le choix de débuter notre corpus à l'année 1992 est lié à la fois à l'arrivée d'Une Famille formidable, mais aussi à un tournant dans la production sérielle française qui, suite à l'imposition de quotas de production, connaît une croissance 
est que ce corpus, qui s'étend sur vingt ans, promeut une figure relativement consensuelle de la mère de famille, voire de la "bonne " mère de famille. Cette dernière est caractérisée par un certain nombre de devoirs ou d'impératifs, qui nous semblent montrer à quel point ces normes sont révélatrices de rapports de domination. En effet, les normes de parentalité sont porteuses d'injonction à bien faire, à adopter certaines pratiques. Tout un dispositif d'institutions est d'ailleurs en place pour s'assurer que les parents jouent bien leur rôle - les sagesfemmes et puéricultrices dès le séjour en maternité, les pédiatres, les instituteurs et institutrices qui sont encouragés à être vigilants. Le poids de ces normes est également lisible par exemple dans " les différentes propositions à tentation répressive qui animent le débat public, comme la suspension des allocations familiales, l'engagement de la responsabilité pénale des parents pour les infractions commises par leurs progénitures, l'obligation, en cas de manquement constaté, de suivre des stages de formation ou de rééducation parentale ${ }^{15}$. On voit bien dans ce type de mesure le poids des normes et la soumission qu'elles impliquent. Afin d'analyser ces normes de parentalité et la nécessité de s'y plier, nous nous appuierons sur la proposition de Danilo Martucelli de définir la domination comme un rapport social renvoyant à la fois à une forme de subordination et à " un complexe d'associations ou de mécanismes assurant la formation et la reproduction du consentement des dominés par une série de compromis divers ${ }^{16}$. Ainsi, nous étudierons les normes de la maternité en les analysant depuis le prisme du consentement implicite à la domination. Pour ce faire, nous resserrerons la focale sur le phénomène de culpabilisation des mères et sur le traitement de la grossesse des personnages. En effet, les femmes enceintes sont soumises à tout un faisceau de normes auxquelles il leur est bien difficile de résister. Ces deux étapes nous permettront de définir les contours de la maternité idéale, telle que définie par la fiction. Pour autant, les séries télévisées ne sont pas uniquement vectrices d'une vision uniforme ou standardisée de la maternité et de ses enjeux, et des espaces de résistance ou de prise de distance à ces normes existent et méritent que l'on s'y attarde. La série Vous les femmes tend ainsi à mettre en scène des mères transgressives, voire déjantées, en détournant les codes de la "bonne " maternité tout en jouant avec ceux de la féminité. Nous nous focaliserons ici sur les saynètes traitant de l'exercice de la parentalité féminine ou de la grossesse, de manière à mettre en évidence les résistances à la domination dont la fiction peut être porteuse.

importante. À ce corpus principal s'ajoute un corpus secondaire, composé de séries mettant en scène des personnages de mères qui nous ont semblé mériter d'être analysés comme ceux de Vous les femmes (M6, 2009 et 2010) ou Alice Nevers, dans la série éponyme de TF1 à partir du moment où elle devient enceinte en 2009.

15 Jacques Faget, "La fabrique sociale de la parentalité », in Alain Bruel et al. (éd.), De la parenté à la parentalité, Toulouse, ERES, 2003, p. 78.

16 Danilo Martuccelli, "Figures de la domination ", Revue française de sociologie, n 3, vol. 45, 2004, p. 469-497, p. 469. 


\section{Les configurations normatives de la maternité dans les séries familiales}

Les héroïnes des séries de notre corpus présentent un certain nombre de caractéristiques communes, elles ont des inquiétudes similaires et surtout toutes accordent une grande importance à leur rôle ou "métier » de mère ${ }^{17}$. La récurrence de pratiques ou de conceptions de la maternité nous a amenée à faire émerger un modèle de mère, modèle que nous aimerions exposer rapidement ici.

\section{Une figure consensuelle : la mère de famille idéale, selon les séries}

Afin de mettre en lumière ce modèle, nous mobilisons l'idéal-type, tel qu'utilisé chez Weber. Rappelons que cet idéal-type ne correspond pas au type moyen, mais qu'il représente davantage un cas idéal, limite. Cet outil nous permet de mettre en avant les caractéristiques réunies par une majorité de personnages-mères, et donc de définir l'idéal auquel tendent la plupart des mères de séries. Cet idéal-type renvoie à quatre déterminants : l'impératif de communication, l'impératif de responsabilité, l'impératif d'amour et l'impératif de réflexivité. La communication semble presque érigée en valeur dans les séries tant est constamment réaffirmée la nécessité de parler et de s'écouter au sein de la famille. Ce sont d'ailleurs souvent les mères qui encouragent au dialogue familial : l'une propose une "opération convivialité " à sa famille ${ }^{18}$ tandis que d'autres insistent pour que leurs enfants ôtent leurs écouteurs à table ${ }^{19}$. L'impératif de responsabilité va de pair avec l'investissement des parents dans les études, mais aussi dans le bien-être de leurs enfants : l'épanouissement de l'enfant est essentiel et c'est le rôle des parents d'y veiller. Ils ont en effet pour devoir, comme le dit François de Singly, d'accompagner l'enfant et de l'aider à devenir lui-même ${ }^{20}$. L'impératif d'amour renvoie au fait que, dans les séries, les mères aiment forcément leur(s) enfant(s). Elles doivent être affectueuses, tendres, aimantes. Les femmes qui ne le sont pas sont stigmatisées et considérées comme déviantes. Coline Cardi, dans son article sur la figure maternelle dans la justice des mineurs, montre bien que l'amour maternel est la norme, et que les mères n'étant pas suffisamment affectueuses et aimantes sont vite qualifiées de " dangereuses". Ainsi, dans Famille d'accueil, Marion demande à Claude - dont la fille a été placée par l'ASE - s'il lui " reste encore deux doigts de sentiments maternels $»^{21}$. En même temps que ses sentiments, elle remet en

17 Nous reprenons ici une expression de Séverine Gojard. Voir Séverine Gojard, Le métier de mère, Paris, La Dispute, 2010.

18 Valérie dans Que du bonheur!, épisode 15, "Opération convivialité », TF1, 25 janvier 2008

19 Sophie dans Hard, épisodes 1, 2 et 3, Canal +, le 9 mai 2008 ou Isabelle dans Merci, les enfants vont bien, épisode " Un nuage passe ", M6, 29 octobre 2008.

20 François de Singly, Comment aider l'enfant à devenir lui-même ?, Paris, Pluriel, 2010 (1 ${ }^{\mathrm{re}}$ éd. 2009).

21 Famille d'accueil, épisode 59, « Une petit héroïne », F3, 7 février 2012. 
cause la capacité de Claude à être une mère pour sa fille Vanessa. Elle encourage en effet Claude - qui est droguée, en couple avec un homme violent et risquant de perdre son emploi - à laisser partir sa fille en internat, c'est-à-dire à la laisser vivre sans elle, pour son bien. La mère malade ou en situation de faiblesse est ainsi stigmatisée et bascule du côté de la « mauvaise » mère, celle qui n’aimerait pas suffisamment son enfant et serait incapable de prendre soin de lui. Enfin, l'impératif de réflexivité renvoie au retour que les mères effectuent sur leurs pratiques parentales. Elles s'interrogent sur leurs façons de faire, craignent de commettre des erreurs, puisent dans toutes sortes de ressources pour être des mères idéales. Ainsi, les héroïnes font appel aux "discours psy "22, et ce recours se fait sous trois formes principales dans les séries : la consultation de sources écrites de vulgarisation psychologique (livres ou magazines) ; la consultation d'un psychologue ou pédopsychiatre ; enfin, l'emploi par les personnages des " discours psy " comme énonciation de règles de parentalité. Dans le premier cas, les mères cherchent des ressources pour exercer au mieux leur rôle de parent : nombreuses sont celles qui achètent des livres traitant d'une question spécifique : Marion Ferrière ${ }^{23}$ lit Le Suicide, Alice Nevers ${ }^{24}$ lit Mère calme, enfant calme, tandis que de son côté Valérie Bouley lit des magazines dans lesquels elle trouve des solutions aux conflits qui l'opposent à sa fille. Valérie d'ailleurs, particulièrement férue de psychologie, va même jusqu'à « consulter » en famille. Le recours aux psychologues prend diverses formes dans la fiction : Catherine Beaumont ${ }^{25}$ en fait une démarche personnelle, un moment à soi durant lequel elle s'autorise à se plaindre de sa vie de famille; dans le cas de Valérie, c'est un moyen d'obtenir des réponses et des solutions. Marion s'inscrit dans cette même démarche : elle demande des conseils aux pédopsychiatres et n'hésite pas à consulter des spécialistes pour soigner les enfants qu'elle accueille.

Le fait que les mères soient en quête de repères ou d'assistance est loin d'être négligeable : cette récurrence du recours à la psychologie montre que la maternité et son exercice nécessitent une sorte de caution ou de garantie qu' elle est bien appliquée, qu'elle se fait, en quelque sorte, dans les règles et que l'enfant est bien encadré, entouré, pris en main. Ces fictions véhiculent l'idée que les problèmes personnels peuvent être résolus grâce à des discours dépassant les cas individuels pour trouver un écho auprès de toutes les mères. Et donc, qu'il y aurait de bonnes façons de faire, au-delà des différences de configuration familiale, de classe sociale ou encore de situation professionnelle. Le dernier fait marquant dans nos fictions est la reprise des " discours psy " par les personnages eux-mêmes. Nous développons ici un exemple issu de la série

22 Nous reprenons ici l'expression de Dominique Mehl. Voir Dominique Mehl, La bonne parole. Quand les psys plaident dans les médias, Paris, Éditions de la Martinière, 2003.

23 Héroïne de Famille d'accueil (F3, depuis 2001).

24 Hérö̈ne éponyme d'Alice Nevers, le juge est une femme (TF1, depuis 2002).

25 Héroïne d'Une Famille formidable (TF1, depuis 1992). 
courte de TF1 Que du bonheur !, diffusée en access prime time en 2008, mais nous procéderions au même type d'analyse dans des séries d'autres formats. Dans l'épisode "Dessin d'école "26, Valérie confie ses inquiétudes à son mari Jean-François à propos de leur fille Zoé. Celle-ci a fait plusieurs dessins en noir et marron et Valérie craint qu'elle soit triste ou malheureuse et qu'elle exprime ce mal-être par le dessin. Elle dit à son mari : " c'est un dessin tout noir et tout marron. Ça m’inquiète. Ça me fait peur Jean-François »; « il faut écouter les dessins des enfants, tous les psys le disent. Demande à ton ex, tu verras. [...] Elle est psy, et elle te confirmera que les enfants s'expriment par les dessins " ou encore " elle nous parle, là, Zoé. Faut qu'on l'écoute». À chaque fois, Jean-François refuse son interprétation et ne s'inquiète pas du tout, lui répondant même que "c'est des conneries". Au final, les parents finissent par comprendre que si Zoé dessine en noir, c'est parce qu'elle n'a pas d'autres crayons de couleur. Cet épisode est intéressant car il témoigne de différentes choses. Tout d'abord, il montre bien que ce sont en général les mères qui s'inquiètent pour les enfants, et qu'elles convoquent, pour appuyer leurs craintes, les « discours psy ${ }^{27}$. La principale solution proposée par Valérie réside dans la communication et l'écoute de l'enfant. L'inquiétude de la mère est finalement hors de propos : elle avait tort de s'inquiéter et, comme le pensait le père, tout va bien. La convocation des " discours psy " est ici tournée en ridicule. Cependant, il est intéressant que ce soit le personnage de la mère qui fasse appel à ces discours et qui s'inquiète de potentiels signes avant-coureurs d'un problème : ce sont ici des caractéristiques « maternelles » qui apparaissent et qui nous rappellent les travaux de Carol Gilligan et l'idée d'une existence de compétences féminines de $\operatorname{care}^{28}$. Dominique Mehl montre bien que ces discours proposent une vision spécifique de la parenté : "le message proféré par les psys appelés à la barre des médias retentit toujours des mêmes accents. Il demeure guidé par cette quête des conduites, valeurs et comportements susceptibles de définir le bon parent, ou plutôt le "presque" bon parent ou le "quasi" bon parent. La grille de lecture de la parentalité moderne par les psys véhicule toujours cette image du bon exercice d'une fonction, d'une bonne adéquation à un rôle $»^{29}$. Comme le souligne la sociologue, la frontière est mince entre le conseil et le devoir, entre la prescription et l'instruction. Nous pouvons noter enfin que, comme bien souvent, ces discours s'adressent essentiellement aux mères, comme si les pères n'avaient guère besoin de

26 Que du bonheur !, épisode 8, "Dessin d'école ", TF1, 16 janvier 2008. Les citations suivantes proviennent de cet épisode.

27 Valérie, dans Fais pas ci, fais pas ça ou Adèle, dans Clash (F2, 2012) procèdent exactement de la même manière.

28 Voir Carol Gilligan, Une voix différente. Pour une éthique du care, traduit de l'anglais par Annick Kwiatek, Paris, Flammarion, 2008.

29 Dominique Mehl, La bonne parole. Quand les psys plaident dans les médias, op. cit., p. 31-32. 
conseils ou de ressources extérieures pour penser leur paternité. Cette convocation des discours psy et la circulation de ces derniers dans la fiction nous semblent révélatrices à la fois de l'investissement des mères dans leur rôle parental et d'une forme de normativité liée à la valorisation de certaines pratiques. En effet, si ce sont essentiellement les mères qui s'inquiètent, ce sont aussi elles qui culpabilisent lorsqu'elles pensent avoir raté quelque chose ou avoir fait une erreur. Leur quête de perfection va dès lors de pair avec une remise en question très forte, qui s'accompagne de culpabilité. Les mères se sentent coupables dès qu'un problème survient. Des expressions comme " c'est de ma faute ", " je suis nulle " ou "j'ai tout raté " sont légion ${ }^{30}$. Les normes d'une bonne maternité, si elles ne sont pas respectées, sont source de culpabilité mais aussi d'angoisse ou de stress pour les mères. Elles consentent implicitement à ces normes et acceptent de s'y plier, quelle que soit la difficulté pour y parvenir. C'est ce qui nous amène à considérer que ces mères sont d'une certaine manière en position dominée puisqu'elles doivent se soumettre à des injonctions parfois difficiles à mettre en œuvre. Ces différents impératifs renvoient à un rôle maternel extrêmement normé et à une image consensuelle de ce que serait, aujourd'hui, une "bonne " mère. En mettant en scène un nombre si important de mères qui s'inquiètent de leurs pratiques et cherchent à " bien " faire, la fiction véhicule l'image d'une mère de famille prenant ce rôle à cœur et surtout d'un rôle engendrant des pratiques spécifiques qui s'imposent aux mères. Ce schéma récurrent sur les vingt années de notre corpus, et quelles que soient les chaînes de diffusion, permet de voir émerger une figure de mère hégémonique dans la fiction.

\section{La grossesse et les ressorts du consentement}

Au cours de notre analyse sur les représentations de la maternité, nous nous sommes intéressés aux représentations de la grossesse. L'un des intérêts de la sérialité est de mettre en scène des personnages dans un temps beaucoup plus long et dilué que celui autorisé par un téléfilm ou un film. Les séries prennent de plus en plus le temps d'exploiter la grossesse d'un personnage principal et d'en faire un ressort narratif. Il nous semble que ce phénomène est assez récent, dans la mesure où notre exploration des archives de l'Inathèque ne nous a pas fait rencontrer beaucoup de femmes enceintes sur une durée

30 Voici quelques exemples de répliques de mères : "D’abord si Nicolas il n’a pas son bac, ce sera de ma faute. Et toi aussi si tu échoues ce sera de ma faute. [...] J'ai tout raté, tout "; « je n'ai pas été à la hauteur » - Catherine dans Une Famille formidable, respectivement épisode 1, "Les parents disjonctent ", TF1, 17 septembre 1992, et épisode 11, " Panique à bord ", TF1, 29 mai 2000 ; "Tout bien réfléchi, depuis que votre père est parti, j'ai tout raté [...] à chaque ratage, c'est vous qui payez l'addition " - Isabelle, dans La Smala s'en mêle, épisode 2 "Sauvage concurrence ", F2, 24 octobre 2012. 
de plusieurs épisodes et semble pouvoir s'expliquer par le fait que la plupart des grossesses étant aujourd'hui désirées ou au moins choisies, l'importance accordée à l'enfant et à ce moment particulier n'a fait que croître. La fiction sérielle tend donc à prendre davantage le temps de traiter les grossesses de ses héroïnes. À titre d'exemple, en 1992, Catherine Beaumont, l'héroïne d'Une Famille formidable apprend qu' elle est enceinte durant le deuxième épisode et la grossesse ne dure que le temps du troisième épisode (pendant la dernière scène du troisième épisode, Catherine révèle qu'elle attend des jumeaux, et dans la première scène de l'épisode quatre les bébés se tiennent assis seuls). Il n'y a aucune exploitation sérielle de cette grossesse dont l'évolution physiologique ne fait absolument pas partie de l'intrigue. Dix-sept ans plus tard, en 2009, la grossesse d'Alice Nevers est traitée sur onze épisodes, dont le dernier au sein duquel elle accouche, tandis que la grossesse de Charlotte, en 2011, dans Famille d'accueil est traitée sur huit épisodes. La fiction sérielle tend donc à prendre davantage le temps de traiter les grossesses de ses héroïnes. Le temps où les grossesses sont traitées par l'ellipse est révolu. Les fictions insistent sur l'idée que les femmes enceintes, soumises à de fortes variations hormonales, ont des comportements pour le moins étranges: Morgane, héroïne de Vive la colo sur TF1, pleure et commente, comme pour s'en excuser : "ça doit être les hormones " ${ }^{31}$, Marion fait de même $:$ " Je [ne] pleure pas, je ris, et puis c'est les

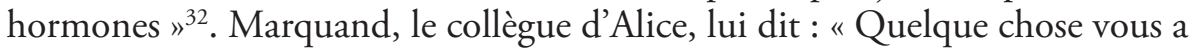
mis de mauvaise humeur ? Je vous sens très hormonale, là " puis à la fin de la conversation : "Dans ce cas il va falloir surveiller vos sautes d'humeur, vous voyez ? "33. On peut noter que Sophie, dans Hard, se révolte contre ce type de remarques ou d'arguments. Après une énième remarque sur ses réactions, elle rétorque : " alors, vous allez arrêter avec vos histoires d'hormones, je vais très bien. Je ne suis pas du tout à cran $»^{34}$. Les femmes enceintes, pour les autres personnages, ne seraient donc pas " dans leur état normal ", la grossesse les transformant en des êtres différents, voire à part, et perdant une part de leur autonomie.

En effet, les femmes enceintes deviennent l'objet d'attentions constantes. Les membres de leur entourage leur font de multiples recommandations de prudence : elles doivent faire attention en voiture, ne pas monter sur une chaise, ne pas se pencher, se reposer, prendre du magnésium, rester allongées... La liste des conseils est longue, nous l'arrêterons là. Les « discours psy » reviennent également en force lorsqu'il s'agit de tracer les frontières des " bonnes " pratiques maternelles et ravivent un certain nombre d'injonctions

31 Vive la colo, épisode 4 «Les Olympiades », TF1, 23 avril 2012.

32 Famille d'accueil, épisode 43, "Hors jeu ", F3, 4 janvier 2011.

33 Alice Nevers, le juge est une femme, épisode 23 « Faute d'ADN », TF1, 4 juin 2009.

34 Hard, saison 2, épisode 12, Canal +, 30 juin 2011. 
liées à la maternité : à plusieurs reprises est rappelée l'idée que le fait de stresser la mère stresserait le bébé, que le père doit parler à son enfant encore dans le ventre de sa mère ${ }^{35}$. À Clem, qui dit de son bébé que "c'est un étranger " et qu'elle n'en veut pas, sa mère lui répond : "faut que t'arrêtes avec ça. Un bébé dans le ventre de sa mère, ça ressent tout ». Elle rappelle également à sa fille : " pas d'alcool, pas de cigarettes, pas de bêtises. [...] Je [ne] te flique pas, c'est sérieux, tu es enceinte. Tu dois faire très attention à toi. À toi, et au bébé ${ }^{36}$. Ces conseils, remarques et interdits - qui s'appliquent à toutes les femmes enceintes, quel que soit leur âge ou leur position sociale - sont le signe d'une infantilisation généralisée de la femme qui, dès lors qu'elle est enceinte, semble avoir perdu toute capacité de jugement. Plus encore, comme l'écrit Barbara Duden, le corps de la femme enceinte ne lui appartient plus. Elle évoque la "manière dont le foetus, une fois admis, dépossède la femme de son corps et la dégrade au rang d'une cliente qui a besoin non seulement d'assistance, mais de conseils $»^{37}$. La plupart des femmes enceintes subissent les remarques, conseils, voire interdictions de leur entourage. Si elles obéissent parfois, acceptant de modifier leur mode de vie pour le bien-être de leur enfant à naître, nombreuses sont celles qui se rebiffent. Ainsi, de plus en plus, les femmes enceintes se rebellent et finissent par dire qu'elles en ont assez, à l'instar d'Alice, qui dit : " tout le monde s'inquiète, me donne des conseils, ça m’étouffe moi !" ou encore de Christine qui dit à son mari : "Sébastien, tu vas pas recommencer? Hier, [il ne] fallait pas que je nage, avant-hier, [il ne] fallait pas que je joue au volley, l'autre jour je marchais trop longtemps et aujourd'hui c'est le soleil! Je [ne] suis pas malade, je suis enceinte ! ${ }^{38}$. Il est d'autant plus intéressant de noter que, si les hérö̈nes refusent d'être, en quelque sorte, maternées, elles acceptent tout de même de nombreuses contraintes liées à leur grossesse. Séverine Gojard rappelle le poids des normes de la maternité sur les mères : "ne pas appliquer les prescriptions, écrit-elle, c'est faire courir à son enfant un risque potentiel $»^{39}$. En effet, la " police des mères $»^{40}$ commence dès la grossesse. La femme enceinte doit se soumettre à tout un faisceau de normes, son corps ne lui appartient plus tant la priorité est donnée à la cause des enfants ${ }^{41}$. La soumission des héroïnes de séries à ces

35 Chloé, dans Ma femme, ma fille, deux bébés, dit ainsi : "j’ai lu sur un forum de femmes enceintes que c'était hyper important pour le bébé d'entendre la voix de son père avant de s'endormir " (épisode pilote, M6, 20 octobre 2010).

36 Clem, épisode pilote, TF1, 22 février 2010.

37 Barbara Duden, L’invention du foetus. Le corps féminin comme lieu public, traduit de l'allemand par Jeanne Étoré, Paris, Descartes et Compagnie, 1996, p. 11.

38 Une Famille formidable, épisode 17, "L’enfer au paradis ", TF1, 11 janvier 2006.

39 Séverine Gojard, Le Métier de mère, op. cit., p. 155.

40 Marcela Iacub, L'empire du ventre. Pour une autre histoire de la maternité, Paris, Fayard, 2004.

41 Voir Sandrine Garcia, Mères sous influence. De la cause des femmes à la cause des enfants, Paris, La Découverte, 2011. 
normes témoigne de leur circulation dans la sphère publique. Plus encore, c'est le caractère prescripteur des médias que l'on peut relever ici. Ils ont en effet la possibilité tout à la fois de discuter certaines normes tout en en renforçant d'autres. Patricia Legouge montre cette ambivalence dans son travail sur le magazine Jeune \& Jolie lorsqu' elle écrit que «si, parfois, les articles concernant la sexualité viennent ébranler certaines catégorisations [...], c'est aussi pour en ériger ou en renforcer d'autres de façon plus insidieuse " ${ }^{42}$. Les médias se font alors le relais d'un certain nombre d'injonctions qui mettent leurs récepteurs (mais plus encore réceptrices) en situation de docilité, voire de soumission.

La maternité semble ainsi parfaitement s'intégrer dans ce mélange de subordination et d'assentiment à la subordination qui compose la domination selon Danilo Martuccelli. Les femmes enceintes sont constamment rappelées à leur devoir et à leur "ventre ». La soumission aux normes va ici de pair avec un consentement implicite à une forme de domination. Les femmes enceintes doivent accepter de modifier leur alimentation, les attentions et conseils de leur entourage, mais aussi de se plier à la volonté du corps médical. La priorité doit être donnée à l'enfant à naître, et non pas à la mère qui doit tout entière se consacrer à cet être en devenir. Béatrice Jacques écrit à ce propos : «l'objectif est que les comportements subjectifs correspondent aux normes biomédicales. Les "bons comportements" d'une femme enceinte sont parfaitement socialisés, les femmes se sentent alors "obligées" de les adopter " ${ }^{43}$. Si les personnages adoptent ces normes, cela ne les empêche pas pour autant d'en dénoncer le caractère contraignant, pour ne pas dire coercitif. La révolte gronde et tend à se faire entendre de manière parfois beaucoup plus virulente. En tant que " médiacultures ${ }^{44}$ ", les séries télévisées sont loin de proposer une vision unifiée du monde, et multiplient les tensions en rendant les contradictions apparentes. À la suite d'Éric Macé, nous considérons ainsi la télévision comme partie prenante d'une sphère publique entendue comme une " arène de la conflictualité sociale et symbolique ${ }^{45}$. La figure consensuelle de mère de famille décrite précédemment nous semble donc être davantage une figure hégémonique plutôt que dominante. En effet, cette figure renvoie à une visée hégémonique car elle est sans garantie et en constant conflit avec d'autres représentations. C'est à ces figures contre-hégémoniques que nous proposons de nous intéresser désormais.

42 Patricia Legouge, "Les représentations de la sexualité dans Jeune et Jolie ", Le Temps des médias, $\mathrm{n}^{\circ} 21,2013$, p. 68-69.

43 Béatrice Jacques, Sociologie de l'accouchement, Paris, PUF, 2007, p. 17.

44 Éric Maigret et Éric Macé, (éd.) Penser les médiacultures. Nouvelles pratiques et nouvelles approches de la représentation du monde, Paris, Armand Colin/INA, 2005.

45 Éric Macé, Les Imaginaires médiatiques. Une sociologie postcritique des médias, op. cit., p. 87. 


\section{Conflictualités, négociations et résistances dans Vous les femmes}

La série Vous les femmes, composée d'une succession de saynètes centrées sur des personnages féminins, prend le contre-pied des représentations habituelles. Ces scènes inversent la représentation consensuelle de la mère de famille. L'une des mères embrasse et cajole un enfant qui n'est pas le sien sans s'en rendre compte ${ }^{46}$, une autre ne se souvient plus de l'endroit où elle a laissé son nourrisson ${ }^{47}$, une dernière perd volontairement sa petite fille pour partir tranquillement en week-end avec son compagnon ${ }^{48}$. C'est ici l'idée même d'un instinct maternel qui est moquée, tournée en dérision. La série montre, dans les pas d'Elisabeth Badinter ${ }^{49}$, que l'amour maternel n'a rien d'évident ou de «naturel », mais que c'est une construction sociale. Elle met également l'accent sur le poids des attendus sociaux relatifs à la maternité en utilisant les ressorts de la parodie ou l'exagération. Ainsi cette série, écrite par un duo de comédiennes qui en sont également les actrices principales, vise à dynamiter les attendus de la féminité, la maternité étant aussi écornée au passage. Lorsque des mères sont en scène, elles transgressent les interdits, ou du moins agissent en décalage avec les attendus de la maternité. Les sketchs fonctionnent sur deux niveaux : une critique implicite de comportements sociaux (notamment genrés) et une transgression des codes sociaux habituels ayant pour effet de rendre les normes implicites visibles et dicibles.

\section{Refuser de consentir : grossesses transgressives}

Prenons l'exemple de la saynète intitulée "La femme enceinte " ${ }^{50}$. Un homme arrive, accompagné de sa femme enceinte, Joëlle, chez des collègues de travail. À peine a-t-il présenté son épouse que chaque collègue se précipite sur elle, sans la saluer, et se focalise sur son corps. Ils touchent alors le ventre de Joëlle ou lui parlent : « coucou là-dedans ! [...] Vous croyez qu'il m'entend ? » dit ainsi Luc.

46 Vous les femmes, épisode 1, «Instinct maternel », M6, 6 juillet 2009.

47 Vous les femmes, épisode 43, "L'heure du biberon », M6, 24 juillet 2010.

48 Vous les fermmes, épisode 45, "Le boulet ", M6, 17 août 2010.

49 Élisabeth Badinter, L'Amour en plus, Paris, Le Livre de Poche, 2010.

50 Vous les femmes, épisode 14, «La femme enceinte », M6, 23 juillet 2009. 

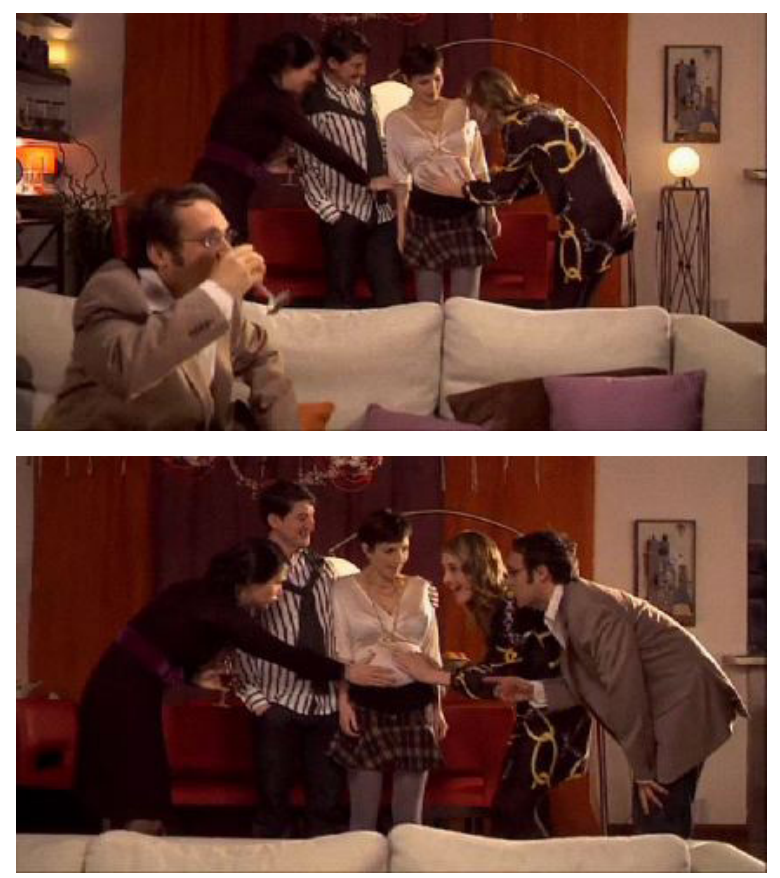

Vous les femmes, épisode 14, M6, photogrammes issus des archives de l'INA.

Gênée, Joëlle se laisse faire, puis se comporte en retour de la même manière. Elle s'approche alors de chacun et, tout comme elle a été touchée sans son consentement, fait de même : elle touche les seins de Géraldine puis les sexes d'Annabelle et de Luc, tout en reprenant les expressions mêmes utilisées par ces interlocuteurs : "c'est tout dur ", " oh mais y'a de la vie, là ", s'exclame-t-elle. Tous trois demeurent estomaqués, tandis que Joëlle sourit. Elle semble agir de façon à se faire accepter, c'est-à-dire en adoptant le même comportement que ses interlocuteurs. Si le fait de poser sa main sur un ventre rond est socialement acceptable dans la scène, le fait de toucher d'une manière similaire des corps "vides " ne l'est pas. Surprise, malaise et désapprobation sont dès lors lisibles sur les visages des autres personnages. 

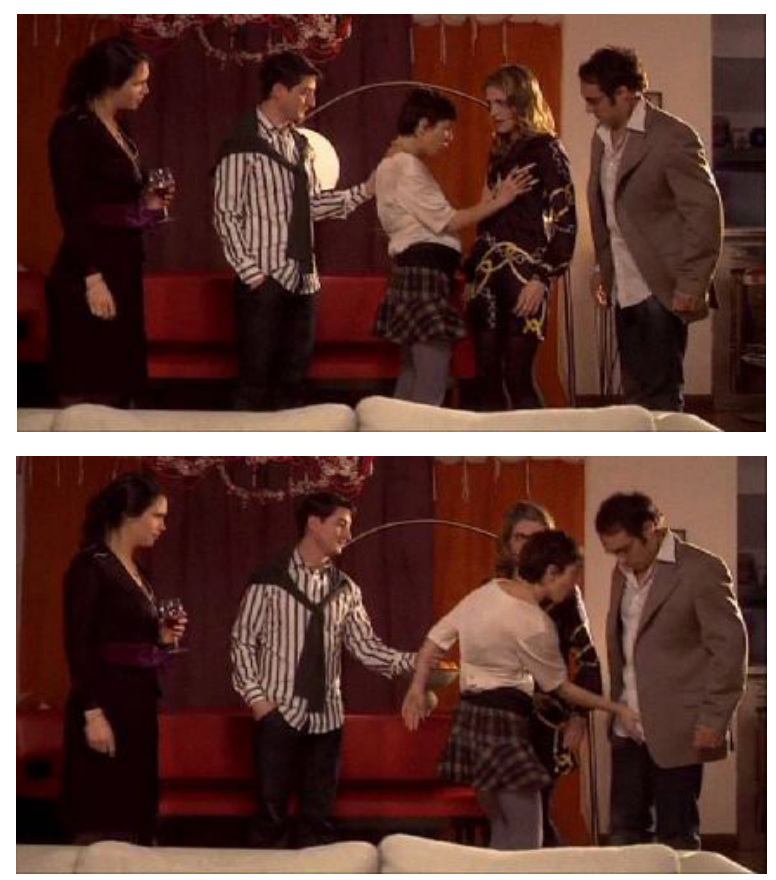

Vous les femmes, épisode 14, M6, photogrammes issus des archives de l'INA.

Les auteures ont choisi d'exagérer encore la situation puisque la femme touche des seins et des sexes mais il nous semble que l'important ne réside pas uniquement dans la partie du corps qui est touchée mais bien dans la transgression d'un contact corporel moins légitime socialement que le premier. L'attitude des personnages est poussée à son paroxysme bien sûr, mais reflète la réalité soulevée précédemment : le corps de la femme enceinte ne lui appartient plus. Le rapport au corps en est bouleversé, chaque personnage s'autorisant à toucher le corps enceint et à vanter ses qualités. Il y a ici une parfaite dissociation entre la femme et le ventre contenant le fotus. La saynète met l'accent sur l'infantilisation de la femme enceinte, et sur le fait que les personnages ne s'adressent guère à elle, mais à son ventre, et donc au bébé. Le contenu du ventre devient, en quelque sorte, prioritaire. La femme enceinte est réduite au stade de contenant, d'utérus. Cette saynète rend selon nous extrêmement lisible, en activant les ressorts de l'humour et de la dérision, la nécessité du consentement implicite de la femme enceinte vis-à-vis de comportements relevant de rapports de domination : il y a une véritable prise de pouvoir sur le corps de la femme enceinte qui d'emblée doit se soumettre à des gestes qui lui déplaisent. 
L'accouchement est lui aussi traité de manière transgressive dans une séquence intitulée "À sa place " ${ }^{51}$. Une femme est en salle de naissance, avec le personnel médical, son compagnon à ses côtés. Ce dernier l'encourage, voire l'invective : "vas-y mon amour, tout va bien, pousse, pousse, pousse, tout va bien ». La femme souffle et souffre et, très vite, décide de montrer à son compagnon l'inconfort de sa position. Elle le force ainsi à se déshabiller et à prendre sa place. Voici un extrait du dialogue :

La femme : Tu arrêtes maintenant, tu retires ton pantalon. Tu retires ton pantalon - il s'exécute - Ton slip. Ton slip ! - il le fait. [...] Tu grimpes sur la table. Tes pieds sur les étriers. Descends au bord de la table. Descends au bord de la table. Voilà. Écarte les jambes. Écarte! Maintenant, tu souffles. Encore. Alors? Tout va bien?

L'homme : Non.

La femme : Alors maintenant tu te tais, tu te tais. Je ne veux plus t'entendre. Tu te tais, tu la boucles. Tu descends. Allez, descends, descends, descends, fous le camp ! Fous le camp ! Je ne veux plus te voir. Laisse-moi bosser.

Cette scène révèle l'intrusion particulièrement forte du corps médical vis-à-vis du corps de la parturiente, qui doit mettre de côté sa pudeur. Béatrice Jacques montre bien à quel point les femmes se soumettent, au nom de l'intérêt de l'enfant à naître, au protocole médical et à ses contraintes ${ }^{52}$. "À sa place » met également en avant les conseils et recommandations des proches, qui ne sont pas toujours les bienvenus. Par ailleurs, en révélant l'inconfort de la position de la femme au moment de l'accouchement, cette saynète peut se lire comme une mise en lumière supplémentaire du déficit d'autonomie de la parturiente. En effet, comme le souligne Béatrice Jacques, « alors qu'on aurait pu penser que dans les services de maternité plus qu'ailleurs, sous l'influence des courants de l'humanisation de la naissance, les femmes disposaient aujourd'hui d'un large espace de négociation et pouvaient plus facilement faire des choix et les imposer, on constate au contraire que la pesanteur du système et de l'idéologie biomédicale limite la capacité des femmes à maitriser totalement leur expérience $»^{53}$. Cette saynète participe d'un mouvement de mise en visibilité des contraintes médicales sur les femmes lors de l'accouchement afin de déconstruire leur évidence. Dans cette série, les femmes luttent contre la domination du corps féminin et refusent de consentir, montrant par là le poids des normes et la transgression que ce refus constitue.

51 Vous les femmes, épisode 40, «À sa place », M6, 3 juillet 2010.

52 Béatrice Jacques, Sociologie de l'accouchement, op. cit, p. 159. Elle ajoute qu' « un double système normatif entoure désormais la prise en charge de la grossesse et de l'accouchement : être une bonne mère, c'est observer strictement les prescriptions du médecin, mais c'est aussi accepter de se consacrer totalement aux besoins de l'enfant (même in utero)" (p. 188).

53 Ibid., p. 159-160. 


\section{Des figures contre-hégémoniques, entre transgression et réaffirmation des normes}

Nous évoquions en introduction la proposition de Séverine Gojard de conceptualiser la maternité comme un " métier ». Elle considère en effet que l'expérience sociale de la maternité passe par un apprentissage et l'acquisition, selon diverses modalités, de compétences et de savoir-faire. Elle renvoie également à un certain nombre de compétences attendues de la part de celles qui seraient des "bonnes " mères. En complément de cette proposition, il nous semble que l'on peut mobiliser ce qu'Everett Hughes appelle des « caractéristiques accessoires attendues $"^{54}$. Ainsi, les mères de notre corpus semblent dotées, globalement, de caractéristiques communes, qui se rapprochent d'ailleurs de qualités : écoute, tolérance, compréhension, tendresse, prise en charge du care au sein de la famille, inquiétude, attitude protectrice. Ces caractéristiques accessoires attendues renvoient à des attentes sociales et à la nécessité pour les mères d'y répondre et de s'y conformer, sous peine d'être reléguées au rang de "mauvaises " mères. Dans Vous les femmes, ces différentes caractéristiques sont détournées ou inversées et, de ce fait, rendues d'autant plus visibles. Ainsi, par exemple, les désirs ou besoins des mères passent avant ceux de leurs enfants qui sont dès lors souvent instrumentalisés - en rupture avec toutes les recommandations de protection de l'enfance ou la norme de l'intérêt supérieur de l'enfant. Une mère habille son bébé d'une tenue fabriquée en serpillère, le " trempe " dans un seau et le laisse ensuite se promener en rampant de façon à ce qu'il nettoie le sol ${ }^{55}$. Dans " promenade familiale " ${ }^{56}$, un couple est dans un parc, un bébé étant dans un porte-bébé, dans les bras du père. La mère s'inquiète de savoir si son bébé est bien : «ça va, il est bien mis son bonnet là ? Elle [n']a pas trop d'air qui passe ? Eh mon bébé! Il [n'] est pas coincé son pied là ? - le père la rassure, elle reprend elle a les mains froides, il fait froid non ? Ça va mon bébé ? Ça va mon cœur ? - au père - allez, c'est mon tour, maintenant ". L'homme rétorque " écoute Marion, je viens juste de la prendre ». Alors que l'on s'attend à ce que la femme demande à porter son bébé, il n'en est rien et l'image suivante nous la montre dans les bras de son compagnon, disant au bébé, désormais sur un banc : "n’aie pas peur mon lapin, papa et maman reviennent tout de suite! ». Le comique naît du "déboîtement des attendus $~^{57}$ dans la mesure où la mère, si elle répond d'abord aux attendus de la maternité, rompt soudainement avec ces derniers. Le rire jaillit de ce comportement, que l'on qualifierait rapidement d'irresponsable,

54 Everett C. Hughes, Le regard sociologique. Essais choisis, textes rassemblés et présentés par JeanMichel Chapoulie, Paris, Éditions de l'EHESS, 1996, p. 188.

55 Vous les femmes, épisode 43, "Maniaque », M6, 24 juillet 2010.

56 Vous les femmes, épisode 10, "Promenade familiale », M6, 17 juillet 2009.

57 Éric Macé, «Rions ensemble des stéréotypes. Anti-stéréotypes humoristiques d'Arabes et de musulmans dans les médiacultures ", Poli, n 2, 2010, p. 17-35, p. 33. 
consistant à laisser un bébé seul sur un banc, dans un parc, en plein hiver. La rupture et la transgression naissent du fait que le plaisir de la mère passe ici avant les besoins de l'enfant. Les mères se comportent donc à l'exact opposé de ce que font leurs consœurs des séries familiales évoquées précédemment. Prenons un dernier exemple. Dans la saynète " Mauvaise école " ${ }^{58}$, une mère est à table avec son fils et sa fille. Le petit garçon lui fait sentir sa compote, dans sa cuiller, et la met sur le nez de sa mère au moment où elle se penche. Le premier réflexe de la mère est de gronder son garçon. Puis elle se prend au jeu et trouve que «c'est vrai qu' elle sent bizarre cette compote ". Elle fait alors sentir son assiette à sa fille, et, au moment où celle-ci se penche, appuie sur sa tête. La mère explose alors de rire, devant les deux enfants - dont la petite fille au visage couvert de compote complètement estomaqués. Ici, la mère se moque clairement de son enfant et les rôles semblent presque inversés. Jamais nous ne verrions Marion Ferrière ou Julie Lescaut écraser la tête de leur enfant dans leur assiette pour leur faire une blague. Ce comportement va à l'encontre des représentations habituelles qui ne mettent jamais en scène une mère se moquant aussi explicitement - presque méchamment - de son enfant. On a donc bien ici une figure de résistance rompant avec les attendues tendresse, douceur ou gentillesse toutes maternelles voire féminines. La série et les personnages qu'elle met en scène fonctionnent ainsi sur le ressort de l'inversion, du décalage, de la rupture avec des pratiques ou compétences attendues, visant à révéler à quel point ces dernières ne sont guère " naturelles » mais bien socialement construites. Par ailleurs, les difficultés du métier de mère et de la conciliation entre gestion des tâches domestiques et gestion des enfants sont elles aussi rendues visibles, comme en témoignent cette mère qui craque à la énième critique de sa fille sur le contenu du réfrigérateur ${ }^{59}$ ou celle qui a posé son fils sur une poubelle et qui arrive devant l'école, un sac poubelle à la main ${ }^{60} \ldots$

La série Vous les femmes met ainsi en scène des figures contre-hégémoniques, venant concurrencer ou du moins résister à la représentation consensuelle de la mère idéal-typique soulignée précédemment. Il nous semble cependant nécessaire de réintégrer ici le caractère comique de cette série et d'en interroger les enjeux. En effet, la transgression des normes d'une "bonne » maternité dépend ici amplement du désir de faire rire le téléspectateur. Le rire va surgir de la rupture avec ce qui est communément admis comme "normal ", évident. On retrouve ici, en partie, l'esthétique carnavalesque, telle que mise en lumière par Bakhtine et parfois utilisée pour saisir les aspects humoristiques de la culture populaire, et notamment des séries ${ }^{61}$. Bakhtine insiste sur les rituels

58 Vous les femmes, épisode 41, " Mauvaise école ", M6, 10 juillet 2010.

59 Vous les femmes, épisode 40, «Le yaourt », M6, 3 juillet 2010.

60 Vous les femmes, épisode 37, «Sac poubelle » M6, 9 septembre 2009.

61 Voir Jean-Pierre Esquenazi, "Les séries télévisées et l'esthétique carnavalesque ", Cinémas : revue d'études cinématographiques, vol. 23, n 2-3, 2013, p. 175-195. 
d'inversion, de renversement et la "logique des choses "à l'envers" "62. Les personnages prennent ici constamment le contre-pied des attitudes attendues, ce qui provoque le rire ${ }^{63}$. Les sketchs rendent ainsi visibles les normes de la maternité en nous montrant ce que les mères ne peuvent se permettre. Cette esthétique proche de l'esthétique carnavalesque « aide à s'affranchir du point de vue prédominant sur le monde, de toute convention, des vérités courantes, de tout ce qui est banal, coutumier, communément admis ${ }^{64}$. La transgression vise à la révélation. Pour autant, le caractère humoristique de ces scènes et de leurs personnages les différencie radicalement des séries se revendiquant de davantage d'authenticité ou de réalisme comme les séries policières, judiciaires, voire familiales. Dès lors, ce type de représentation n'est imaginable que dans le cadre d'un programme explicitement humoristique. L'absence de ruptures de ce genre dans les séries plus "sérieuses " révèle à la fois la prégnance de notre figure hégémonique et la difficulté de rompre avec les impératifs liés à la maternité. En ce sens, dans quelle mesure et jusqu'à quel point le caractère comique de la série permet-il de rompre avec les normes, ou au contraire les renforce-t-il, en circonscrivant leur remise en question radicale au domaine de l'humour ? Comme l'écrit Umberto Eco, « l'humour ne nous promet pas une libération : au contraire, il nous met en garde contre l'impossibilité d'une libération globale, nous rappelant la présence de la loi à laquelle nous n'avons plus raison d'obéir. En cela, il souligne la loi " ${ }^{65}$. La série Vous les femmes fonctionne ainsi comme un révélateur des normes de la maternité et de l'injonction faite aux femmes de s'y soumettre de bonne grâce, mais révèle aussi l'impossibilité de remettre en cause ces normes en dehors du cadre de l'humour affiché et de la dérision revendiquée.

\section{Conclusion}

Les séries familiales construisent une figure de la mère de famille idéale, dont chaque héroïne investit une facette et révèle le caractère normatif. Le désir d'être une "bonne " mère ou de se conformer à des normes implicites - mais non moins prégnantes - engendre des phénomènes de culpabilisation ou de remise en question très fortes. La maternité, dès lors, devient le lieu d'expression de rapports de domination au cœur desquels le consentement est central. Les représentations de la grossesse permettent de saisir les voies de cette

62 Mikhaïl Bakhtine, L'Euvre de François Rabelais et la culture populaire au Moyen Âge et sous la Renaissance, traduit du russe par Andrée Robel, Paris, Gallimard, 1970, p. 19.

63 Cela peut aussi engendrer une sorte de malaise ou de gêne. Ainsi, rien ne garantit que tous les téléspectateurs rient devant la petite fille au visage couvert de compote.

64 Mikhaill Bakhtine, op. cit., p. 44.

65 Umberto Eco, "The Frames of Comic "Freedom" ", in Umberto Eco, V.V. Ivanov, Monica Rector, Carnival!, Berlin, Mouton, 1984, p. 8 (notre traduction). 
acception et de révéler le nécessaire effacement de la femme/mère au profit de son enfant, de son bien-être et de son épanouissement. Pour autant, ce modèle est concurrencé par des figures contre-hégémoniques qui agissent sur plusieurs niveaux : elles rendent les normes visibles et dicibles, déconstruisent leur caractère d' " allant de soi » et font émerger des discours et représentations résistants. Les fictions sérielles, si elles reconduisent les injonctions pesant sur la maternité, parviennent malgré tout à se saisir de ces contraintes normatives, pour en discuter les contours, en redessiner les frontières et montrer d'autres voies. C'est finalement par le biais de l'humour et de la dérision que les redéfinitions sont possibles, et acceptables. En effet, le rire offre une transgression tout en laissant toujours ouverte la voie de la norme, de l'habitude, de l'allant-de-soi. La fiction humoristique apparait donc comme un bastion de résistance et le lieu toujours ouvert à la négociation des identités.

\author{
Sarah Lécossais \\ Laboratoire CIM, équipe MCPN \\ Université Sorbonne Nouvelle Paris 3 \\ sarah.lecossais@univ-paris3.fr
}

\begin{abstract}
Résumé
Cet article étudie les représentations de la maternité dans les séries télévisées françaises en axant l'analyse sur la circulation des normes relatives à la parentalité et la manière dont les personnages les intériorisent ou au contraire tentent d'y résister. Nous mettons en lumière les configurations normatives de la maternité - avec l'exemple de la grossesse des personnages avant de nous concentrer sur les marges de la fiction comme espaces de résistance.
\end{abstract}

\title{
Mots-clés
}

Séries télévisées, maternité, normativité, hégémonie, contre-hégémonie.

\begin{abstract}
This article aims at analyzing the representations of motherhood and pregnancy in French contemporary TV serials. It studies how the characters deal with norms of motherhood and parenthood and how they resist to them, by mobilizing the concepts of hegemony and counterhegemony as theorized by Stuart Hall.
\end{abstract}

Keywords

TV series, motherhood, social norms, hegemony, counter hegemony. 